\title{
Iatrogenic pseudoaneurysm after bevacizumab therapy in patients with metastatic colorectal cancer: Two case reports
}

\author{
CHING-CHUN LI ${ }^{1}$, HSIANG-LIN TSAI ${ }^{1,2}$, CHING-WEN HUANG ${ }^{1-3}$, \\ YUNG-SUNG YEH $^{1,4,5}$, TZU-HSUEH TSAI ${ }^{6}$ and JAW-YUAN WANG ${ }^{1-4,7}$
}

\begin{abstract}
${ }^{1}$ Division of Colorectal Surgery, Department of Surgery, Kaohsiung Medical University Hospital; ${ }^{2}$ Department of Surgery, Faculty of Medicine, ${ }^{3}$ Graduate Institute of Medicine and ${ }^{4}$ Graduate Institute of Clinical Medicine, College of Medicine; ${ }^{5}$ Division of Trauma, Department of Surgery and ${ }^{6}$ Department of Radiology, Kaohsiung Medical University Hospital; ${ }^{7}$ Center for Biomarkers and Biotech Drugs, Kaohsiung Medical University, Kaohsiung 807, Taiwan, R.O.C.
\end{abstract}

Received January 19, 2018; Accepted April 17, 2018

DOI: $10.3892 /$ mco.2018.1712

\begin{abstract}
Pseudoaneurysms are extremely rare in patients with metastatic colorectal cancer (mCRC) treated with FOLFIRI and bevacizumab in the first-line setting. We herein present two rare cases of iatrogenic pseudoaneurysm developing in patients with mCRC after administration of FOLFIRI and bevacizumab. The first patient was a 57-year-old man who was admitted to our institution with intermittent massive bloody discharge following treatment with the 9th cycle of FOLFIRI combined with bevacizumab. Colonoscopic examination revealed sizeable bloody clots in the rectum, but no active bleeder was identified; however, the patient's hemoglobin level was found to be persistently decreased, so an angiography was performed. The angiography revealed a pseudoaneurysm with contrast extravasation from a branch of the left internal iliac artery. Embolization of the bleeding vessel was performed, and the post-embolization angiography revealed no active bleeding. The second patient was a 65-year-old man who observed blood in the stool following treatment with the 5th cycle of FOLFIRI combined with bevacizumab. The angiography revealed a pseudoaneurysm in the superior rectal artery, and intravascular embolization with coils was performed. The patient was discharged without any signs of recurrent bleeding. Following a review of the relevant English literature, to the best of our knowledge, this is the first report on the formation of a bevacizumab-related pseudoaneurysms in mCRC patients. The aim of this study was to alert clinicians to the possibility of this rare complication.
\end{abstract}

Correspondence to: Professor Jaw-Yuan Wang, Division of Colorectal Surgery, Department of Surgery, Kaohsiung Medical University Hospital, 100 Tzyou 1st Road, Kaohsiung 807, Taiwan, R.O.C.

E-mail: cy614112@ms14.hinet.net; jayuwa@kmu.edu.tw

Key words: metastatic colorectal cancer, bevacizumab, pseudoaneurysm, embolization

\section{Introduction}

Combining conventional systemic chemotherapy with bevacizumab, an angiogenesis inhibitor, is currently recommended as first-line treatment for patients with metastatic colorectal cancer (mCRC), as it achieves a median overall survival of 22.7 months (1).

Bevacizumab was developed by Ferrara $\mathrm{et} \mathrm{al}$ as a recombinant humanized antivascular endothelial growth factor monoclonal immunoglobulin G antibody (2) and it has been approved by the US Food and Drug Administration as an adjuvant agent in the treatment of mCRC. However, bevacizumab therapy has been associated with certain adverse events, such as bleeding (3\%), gastrointestinal perforation (2\%), arterial thromboembolism (1\%), hypertension $(5.3 \%)$, proteinuria (1\%) and wound healing complications (1\%) (3-6). Furthermore, hemorrhagic complications, such as pulmonary hemorrhage and hemoptysis, have been reported, mainly in patients with lung cancer, whereas gingival and vaginal bleeding have also been reported, although less commonly (3). In 2015, a retrospective study by Collins et al (7) reported that bleeding occurred in $41 \%$ of mCRC patients; however, the incidence of grade 3 bleeding was found to occur in only $1.6 \%$ of the cases, and there were no reported cases of grade 4 or 5 bleeding.

We herein present two cases of pseudoaneurysm with massive lower gastrointestinal bleeding in patients with mCRC during treatment with FOLFIRI and bevacizumab.

\section{Case reports}

Case 1. A 57-year-old male patient was diagnosed with rectal carcinoma with liver metastases. The patient had a previous history of hypertension, which was well-controlled with medication, but no previous history of malignancy. Colonoscopy revealed a polypoid tumor with superficial ulceration located $8 \mathrm{~cm}$ proximal to the anal verge (Fig. 1A); biopsy of the rectal tumor was suggestive of moderately differentiated adenocarcinoma. Abdominal computed tomography (CT) scans revealed a rectal tumor (Fig. 1B, circle) with concomitant multiple liver metastases (Fig. 1C and D, arrows) and regional lymph 
nodes metastases; the disease was determined to be clinical stage cT3N2aM1a (stage IVa). Neoadjuvant concurrent chemoradiotherapy (CCRT) was administered in accordance with treatment guidelines, and FOLFIRI [irinotecan $120 \mathrm{mg} / \mathrm{m}^{2}$ as a 120-min IV infusion, leucovorin (LV) $200 \mathrm{mg} / \mathrm{m}^{2}$ as an IV infusion over $2 \mathrm{~h}$, and 5-fluorouracil (5-FU) $2,800 \mathrm{mg} / \mathrm{m}^{2}$ as an IV infusion over a 46-h period biweekly] combined with bevacizumab $(5 \mathrm{mg} / \mathrm{kg})$ was administered as a first-line chemotherapeutic regimen. After a treatment period of 3 months, CT scans were used to evaluate the response to neoadjuvant therapy; the scans revealed simultaneous shrinkage of both the liver metastatic lesion and the primary rectal tumor.

Six days after the 9th cycle of bevacizumab injections, the patient visited our emergency room after having passed a massive bloody clot from his anus. The physical findings on initial presentation included a blood pressure of $124 / 78 \mathrm{mmHg}$, heart rate of 130 beats per min, and hemoglobin levels of $8.6 \mathrm{~g} / \mathrm{dl}$. Contrast-enhanced abdominal CT scans revealed no evidence of gastrointestinal tract or vascular abnormalities (data not shown). The patient received a blood transfusion and was admitted to the hospital with a tentative diagnosis of lower gastrointestinal bleeding.

On colonoscopic examination performed after admission, there was blood in the sigmoid colon and rectum; however, there was no sign of active bleeding in the colon or rectum. The patient's hemoglobin level decreased to $6.2 \mathrm{~g} / \mathrm{dl}$, even after the blood transfusion, and an angiography was scheduled as a further diagnostic measure. The angiography revealed a pseudoaneurysm with contrast extravasation in a branch of the left internal iliac artery (Fig. 2A). Active bleeding from the pseudoaneurysm was observed; therefore, transcatheter embolization was performed. A Tornado coil and Nester coil (both from Cook Inc., Bloomington, IN, USA) were inserted in this branch of the left internal iliac artery, and $\sim 3 \mathrm{ml}$ of Avitene mixture (Davol Inc., Woburn, MA, USA) was infused together with a contrast medium. Post-embolization angiography revealed no signs of further bleeding (Fig. 2B), and the patient's hemoglobin level progressively increased to $9.6 \mathrm{~g} / \mathrm{dl}$. The patient was eventually discharged after 2 weeks of uneventful hospitalization, but he ultimately succumbed to progressive metastatic multiple liver lesion deterioration 1 year after this event.

Case 2. A 65-year-old male patient was diagnosed with rectosigmoid carcinoma and associated liver and lung metastasis. The patient had a previous history of hypertension and type 2 diabetes mellitus, which was well-controlled with medication, but no previous history of malignancy. Colonoscopy revealed an ulcerated tumor located $14-18 \mathrm{~cm}$ proximal to the anal verge (Fig. 3A); biopsy was suggestive of adenocarcinoma. Abdominal CT scans revealed a rectosigmoid tumor (Fig. 3B) with concomitant multiple liver metastases and bilateral lung metastasis; the disease was determined to be clinical stage T4aN2aM1b (stage IVb). CCRT was used in accordance with treatment guidelines, and FOLFIRI combined with bevacizumab was administered as a first-line chemotherapeutic regimen. After a treatment period of 3 months, CT scans were performed to evaluate the response to neoadjuvant therapy; the scans revealed mild progression of the rectosigmoid cancer, with pericolic fat infiltration.
Eight days after the 5th cycle of bevacizumab injections, the patient visited our emergency room after having observed a large amount of blood in the stool. On initial physical examination, the blood pressure was $97 / 66 \mathrm{mmHg}$, the heart rate was 104 beats per min, and the hemoglobin level was $7.8 \mathrm{~g} / \mathrm{dl}$. The patient received a blood transfusion and was admitted to our hospital with a tentative diagnosis of lower gastrointestinal bleeding.

Contrast-enhanced abdominal CT scans revealed a small pseudoaneurysm $(0.5 \mathrm{~cm})$, and an angiography was scheduled as a further diagnostic measure. The angiography revealed a pseudoaneurysm with contrast extravasation in the superior rectal artery (Fig. 3C). Active bleeding from the pseudoaneurysm was observed; therefore, transcatheter embolization with coils was performed. Straight coils ( $2 \times 1$ and $5 \times 1 \mathrm{~mm})$ were inserted in the pseudoaneurysm feeder, and post-embolization angiography revealed no further bleeding (Fig. 3D). The patient was eventually discharged after 2 weeks of uneventful hospitalization, but he eventually succumbed to progressive metastatic multiple liver and lung lesion deterioration 9 months after this event.

\section{Discussion}

Radiological imaging plays an important role in primary diagnostics, staging, evaluation of treatment response, follow-up, and even for minimally invasive interventions. CT and MRI are included in national and international guidelines and structured reporting is strongly recommended (8).

Bleeding complications associated with bevacizumab therapy may be attributed to the anti-angiogenic effect of this agent, which inhibits endothelium growth, thus resulting in vessel wall breach and the formation of pseudoaneurysms. In the present cases, the exact pathophysiological mechanism involved in pseudoaneurysm development remains unclear. However, abdominal CT scans of the patient performed prior to bevacizumab therapy revealed no radiological signs of aneurysm formation.

Angiogenesis inhibitors have rapidly emerged as first-line treatment in cancer therapy, usually in combination with cytotoxic chemotherapy. The most frequent toxicity encountered with the use of bevacizumab is hypertension, which occurs in up to $32 \%$ of the patients (9). Patients with hypertension are most commonly treated with oral agents, such as diuretics or calcium channel blockers; however, a small number of patients do not respond to this treatment, and bevacizumab must be discontinued $(10,11)$. Our patient had hypertension prior to treatment; the condition did not worsen with the concomitant administration of bevacizumab and was well-controlled with the use of antihypertensive medication.

The exact pathophysiological mechanism involved in the development of the pseudoaneurysm in our cases has not been fully elucidated. However, a possible correlation between the injections of bevacizumab and the formation of a common iliac or superior rectal artery aneurysm may be reasonably hypothesized; thus, it cannot be excluded that bevacizumab may have been a potential trigger. The aim of the present study was to alert clinicians to this rare adverse event associated with the use of bevacizumab. 


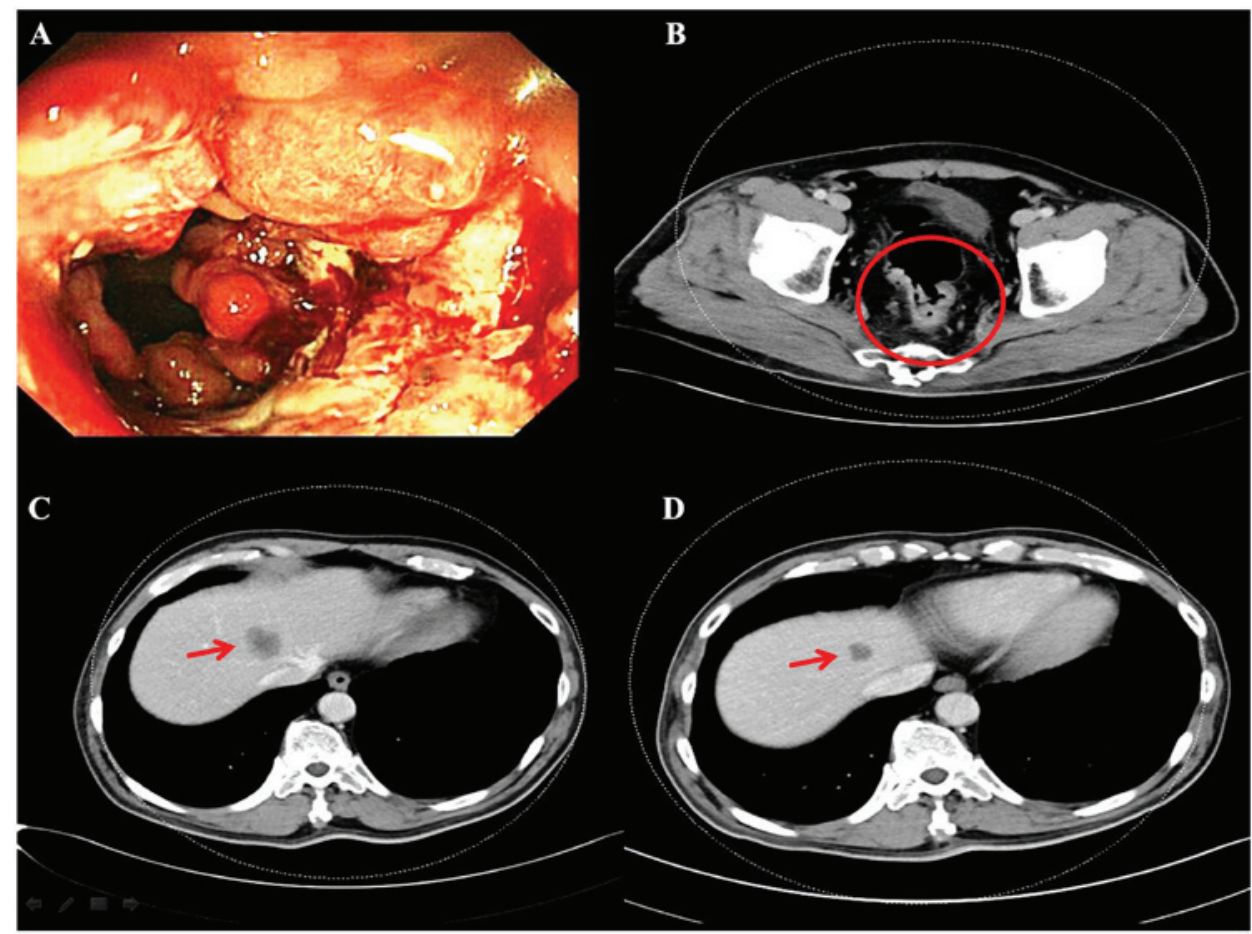

Figure 1. (A) Colonoscopy revealed a lobulated polypoid tumor with superficial ulceration, located $8 \mathrm{~cm}$ proximal to the anal verge. (B) Axial view of abdominal computed tomography (CT) scans showing a lesion in the rectum (circle). (C and D) Axial view of abdominal CT scans showing liver metastasis (arrow).

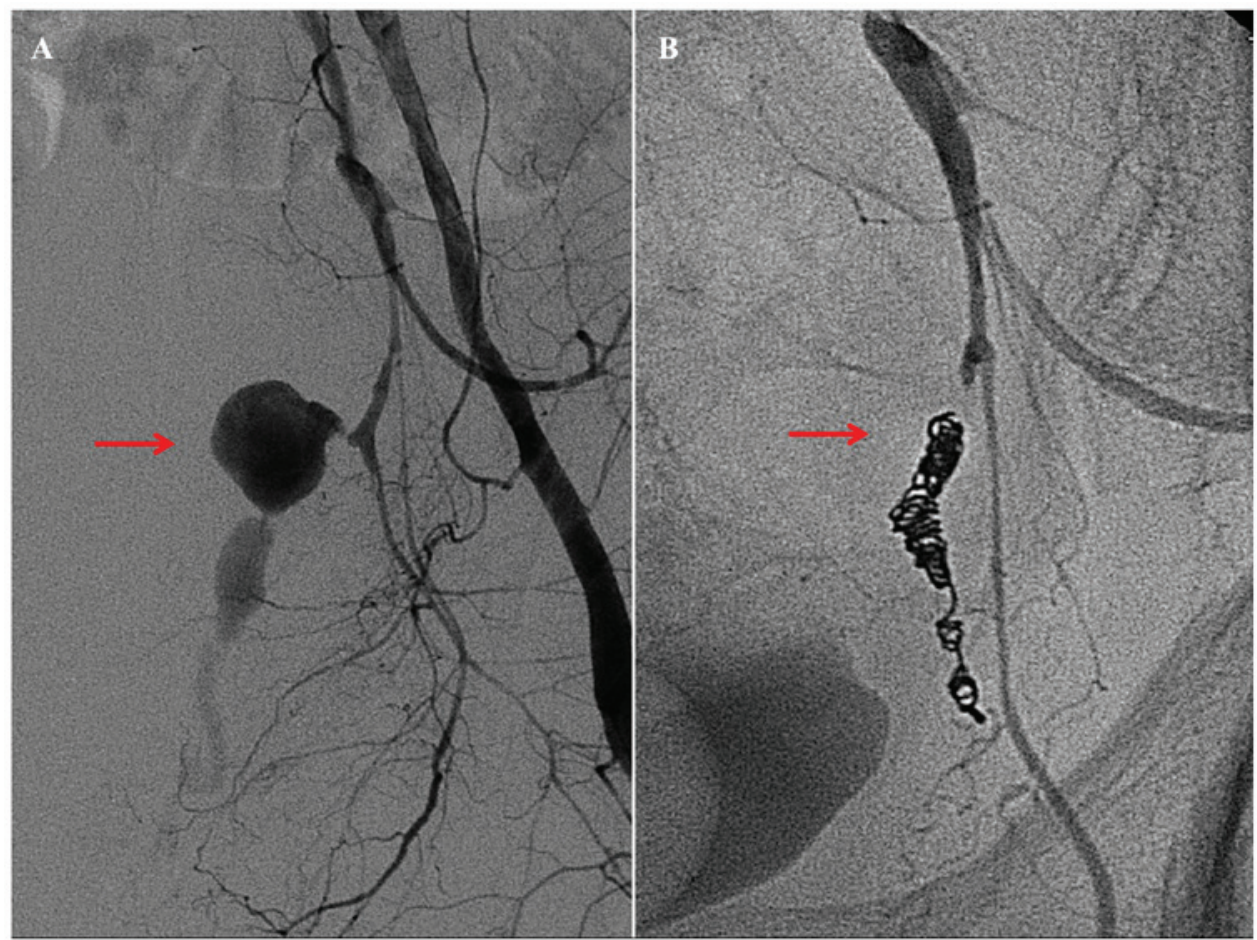

Figure 2. (A) Angiography of the left common iliac artery revealed a pseudoaneurysm with contrast extravasation from a branch of the left internal iliac artery (arrow). (B) No active bleeding was observed post-embolization (arrow).

Based on this observation, it is recommended that patients selected for anti-angiogenic treatment are closely monitored through imaging, and whenever a gastrointestinal massive bleeding from a pseudoaneurysm occurs or a pseudoaneurysm increases in size, it should be managed with selective embolization.
In conclusion, to the best of our knowledge, cases of pseudoaneurysm formation in mCRC patients under bevacizumab therapy have not been previously reported. We herein describe two such cases, and although there is no direct evidence of an association between bevacizumab therapy and the formation of pseudoaneurysms, the need for its consideration during 


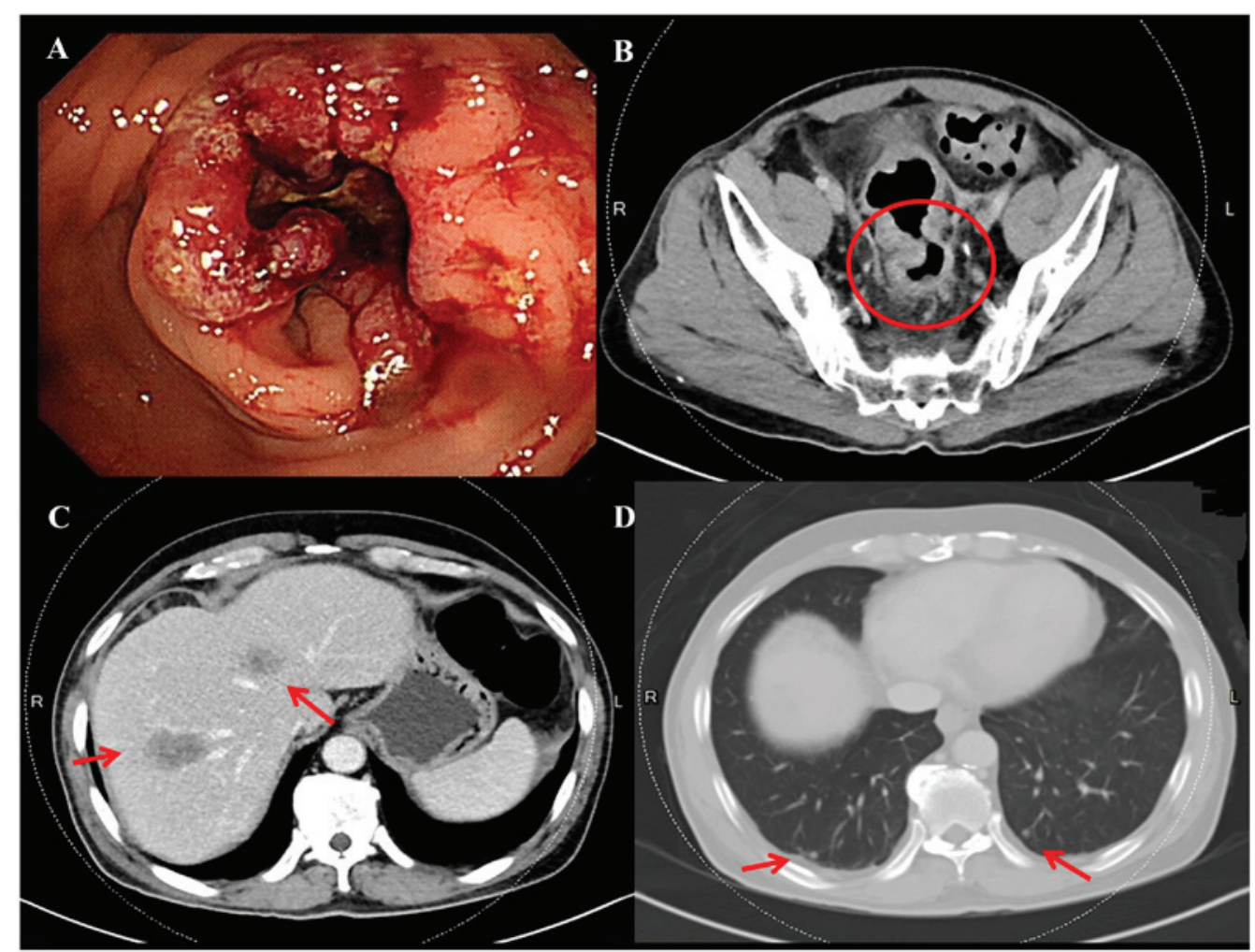

Figure 3. (A) Colonoscopy revealed a ulcerated tumor located 14-18 $\mathrm{cm}$ proximal to the anal verge. (B) Axial view of abdominal computed tomography (CT) scans showing a lesion in the rectum (circle). (C) Axial view of abdominal CT scans showing liver metastasis (arrows). (D) Axial view of abdominal CT scans showing bilateral lung metastasis (arrows).
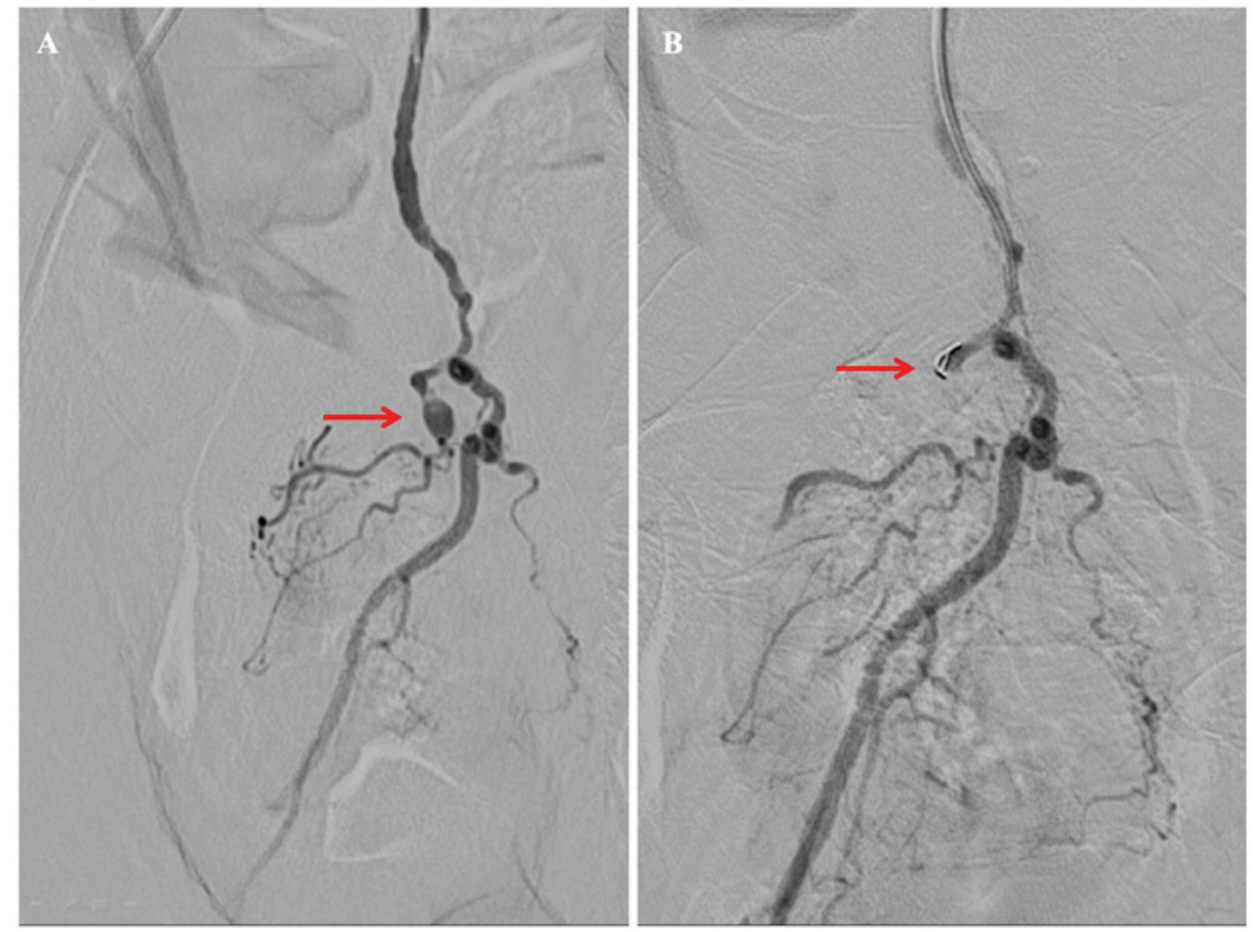

Figure 4. (A) Angiography revealed a pseudoaneurysm with contrast extravasation from the superior rectal artery (arrow). (B) No active bleeding was observed post-transcatheter embolization with coils (arrow).

anti-angiogenic therapy must be emphasized. When a pseudoaneurysm with bleeding becomes apparent and is life-threatening, transcatheter embolization is the preferred treatment.

\section{Acknowledgements}

Not applicable. 


\section{Funding}

The present study was supported by grants through funding from the Ministry of Science and Technology (MOST 107-2321-B-037-003-) and the Kaohsiung Medical University Hospital (KMUHS10601, KMUHS10608, KMUHA10664). In addition, this study was supported by the Grant of Biosignature in Colorectal Cancers, Academia Sinica, Taiwan, R.O.C.; and Grant by the Kaohsiung Medical University aim for the top University, grant no. KMU-S105011.

\section{Availability of data and materials}

Not applicable.

\section{Authors' contributions}

CL was responsible for document recording and manuscript writing. HT, $\mathrm{CH}$ and YY were responsible for image analyses and providing the colonoscopic images. TT was responsible for angiographic diagnosis and treatment. JW was responsible for coordinating with investigators. All authors contributed to reviewing the various drafts of the manuscript and approved the final version of the article.

\section{Ethics approval and consent to participate}

The present study was approved by the Institutional Review Board of Kaohsiung Medical University Hospital (KMUHIRB-20130020).

\section{Patient consent for publication}

The patients have signed an informed consent form regarding the publication of the case details and associated images.

\section{Competing interests}

The authors declare that they have no competing interests.

\section{References}

1. Van Cutsem E, Rivera F, Berry S, Kretzschmar A, Michael M, DiBartolomeo M, Mazier MA, Canon JL, Georgoulias V, Peeters M, et al: Safety and efficacy of first-line bevacizumab with FOLFOX, XELOX, FOLFIRI and fluoropyrimidines in metastatic colorectal cancer: The BEAT study. Ann Oncol 20: 1842-1847, 2009.

2. Ferrara N, Hillan KJ, Gerber HP and Novotny W: Discovery and development of bevacizumab, an anti-VEGF antibody for treating cancer. Nat Rev Drug Discov 3: 391-400, 2004.

3. Gordon MS and Cunningham D: Managing patients treated with bevacizumab combination therapy. Oncology 69 (Suppl 3): S25-S33, 2005.

4. Heinzeling JH and Huerta S: Bowel perforation from bevacizumab for the treatment of metastatic colon cancer: Incidence, etiology, and management. Curr Surg 63: 334-337, 2006.

5. You YN, Wolff BG, Boardman LA, Riegert-Johnson DL and Qin R: Peutz-Jeghers syndrome: A study of long-term surgical morbidity and causes of mortality. Fam Cancer 9: 609-616, 2010.

6. Kesmodel SB, Ellis LM, Lin E, Chang GJ, Abdalla EK, Kopetz S, Vauthey JN, Rodriguez-Bigas MA, Curley SA and Feig BW: Preoperative bevacizumab does not significantly increase postoperative complication rates in patients undergoing hepatic surgery for colorectal cancer liver metastases. J Clin Oncol 26: 5254-5260, 2008.

7. Collins D, Ridgway PF, Winter DC, Fennelly D and Evoy D: Gastrointestinal perforation in metastatic carcinoma: A complication of bevacizumab therapy. Eur J Surg Oncol 35: 444-446, 2009.

8. Baeßler B, Maintz D and Persigehl T: Imaging procedure for colorectal cancer. Visc med 32: 166-171, 2016.

9. Cao D, Guo CH, Liu JW, Yang X and Li Q: Bleeding after bevacizumab treatment in patients with metastatic colorectal cancer. Tumori 101: 46-51, 2015.

10. Kabbinavar FF, Schulz J, McCleod M, Patel T, Hamm JT, Hecht JR, Mass R, Perrou B, Nelson B and Novotny WF: Addition of bevacizumab to bolus fluorouracil and leucovorin in first-line metastatic colorectal cancer: Results of a randomized phase II trial. J Clin Oncol 23: 3697-3705, 2005.

11. Pañares RL and Garcia AA: Bevacizumab in the management of solid tumors. Expert Rev Anticancer Ther 7: 433-445, 2007.

(i) $\Theta$ This work is licensed under a Creative Commons Attribution-NonCommercial-NoDerivatives 4.0 International (CC BY-NC-ND 4.0) License. 